\title{
The Advancements in the Early Detection of Zika Virus Infection
}

\author{
Sharon Sherry Huang ${ }^{1}$, Erick Ceasar Huang ${ }^{2} \&$ Chao Shen Huang ${ }^{3}$ \\ ${ }^{1}$ Department of Biology, Stanford University, Stanford, CA, USA \\ ${ }^{2}$ Department of Neuroscience, Baylor University, Waco, TX, USA \\ ${ }^{3}$ Da Chia Clinic, Taichung, Taiwan \\ Correspondence: Chao Shen Huang, Da Chia Clinic, Taichung, Taiwan. E-mail: cs2777726@gmail.com.
}

Received: July 19, 2018 Accepted: August 22, 2018 Online Published: September 3, 2018

doi:10.5539/gjhs.v10n10p30 URL: https://doi.org/10.5539/gjhs.v10n10p30

\begin{abstract}
The Zika Virus (ZIKV) was propelled to international attention during its outbreak from 2015-2016. Interestingly, the most recent outbreak was not ZIKV's first, although it proved to be the most widespread and impactful, with millions affected in South America, Asia and Africa. Presently no longer considered a global emergency, ZIKV has managed to invoke fear and realization of the susceptibility of the global population to rapidly evolving viruses. In addition, the difficulty of diagnosing the virus demonstrates a deficiency in a rapid, virus specific, and accurate diagnostic tool for the family of flaviviruses that ZIKV belongs to. This paper reviews the approved identification methods along with an analysis of the advantage and disadvantages of each, as well as emerging alternative approaches in ZIKV diagnosis. Common problems with currently utilized methods include slow turnover time, limited throughput, need for further testing to confirm diagnosis, narrow sample compatibility, and cross reactivity to DENV and other similar viruses, Although newer methods discussed in the paper, namely Electrogenerated Chemiluminescence (ECL) and Reporter Virus Neutralization Test (RVNT), show improvement in throughput quantity, speed, and efficiency, it is not certain whether these tests are virus specific and will not react in the presence DENV. The rapidity of diagnosis is important in ensuring timely access to treatment as well as tracking and containing future possible epidemics, Concurrently, virus specificity is equally crucial in ensuring correct diagnosis. Thus, the challenge lies in finding the balance between the two.
\end{abstract}

Keywords: Zika virus, dengue fever, RNA nucleic acid testing, IgM serology test, plaque reduction neutralization

\section{Introduction}

Zika virus (ZIKV) testing from human urine and amniotic fluid samples, as well as that of= chikungunya virus (CHIKV) and dengue virus (DENV) in other specimen was first discovered and isolated in 1947 from a rhesus monkey in the Zika forest in Uganda (Dick, 1952). ZIKV is transmitted to humans via infected Aedes spp. mosquitoes (Haddow, 1964; Wong et al., 2013). Zika virus infections in human are believed to be endemic in Africa and Southeast Asia (Hayes, 2009). In 2007, an epidemic Zika Virus human infections broke out in Yap, Federated States of Micronesia and in the Pacific region, in which less than $70 \%$ of the population were seropositive (Duffy, 2009). In-between 2007-2013, only occasional human infected cases were reported in Africa and Southeast Asia mostly in travellers (Kwong, 2013; Foy, 2011).

The symptoms, if any, of Zika virus infection in humans include low fever, myalgia, retro-orbital pain, headache, conjunctivitis, arthralgia, and skin rashes (Duffy, 2009). Very few cases of victims have been reported to have hemorrhagic signs. A few neurological complications such as Guillain-Barre syndrome have been reported (Kwong, 2013; Foy, 2011). However, most of the infected cases are asymptomatic or with symptoms similar to nonspecific influenza making the acute phase diagnosis of Zika virus infection difficult.

\subsection{Primary Major Outbreak: Micronesia}

Although ZIKV's largest epidemic was in 2015-2016, it was not it's first one. In 2007, a local outbreak of Zika virus human infection was detected on Yap island of Micronesia. Most victims reported rash, arthralgia, conjunctivitis and fever. No hospitalization, hemorrhagic symptoms or death due to Zika virus infections was found. However over $73 \%$ of Yap island residents older than 3 years old were estimated to have been infected with Zika virus. Even without significant symptoms, this demonstrated that transmission of Zika virus in human has occurred outside of Asia and Africa, prompting the awareness of further expansion of Zika virus 
transmissions among the public health officials and clinicians (Duffy, 2009).

\subsection{Detection of Zika Virus Infection}

Only symptomatic patients were reported to have traveled or stayed in the Zika virus endemic areas. Similar clinical symptoms may have occurred in the patients infected by other mosquitoes transmitted diseases such as dengue fever or chikungunya infection (World Health Organization). A quick diagnostic laboratory test is important to differentiate Zika virus infected patients from other virus diseases. The FDA has suggested several diagnostic tools for Zika virus in Emergency Use Authorization (EUA), which includes the Trioplex Real-Time RT-PCR (rRT-PCR) assay and the Zika MAC-ELISA (Center for Disease Control, 2016).

\subsection{Molecular Test for Zika Virus, RNA Nucleic Acid Testing (NAT): Trioplex Real-time PCR}

\subsubsection{Assay Principle}

Trioplex real-time PCR (Trioplex rRT-PCR) assay uses primers and dual-labeled hydrolysis (Taqman ${ }^{\circledR}$ ) probes to quantify viral RNA in human samples. Viral RNA is then reverse transcribed to produce cDNA, which are subsequently introduced to Virus-specific probes that bind to the targeted region of DNA sandwiched between two unlabeled PCR primers. Different fluorescent dyes are used to pinpoint the presence of ZIKV, CHIKV and DENV. PCR amplification allows strand templates to guide Taq polymerase to extend the unlabeled primers. When it reaches the probes, the polymerase cleaves the probes, disengaging them from the quenchers and allowing them to fluoresce.As each PCR cycle progresses, additional probes are cleaved, allowing the quantification of viral RNA to be proportionally correlated with intensity of fluorescence .

Trioplex rRT-PCR can distinguish differences between dengue, chikungunya, Zika viruses in various sample forms, including serum, cerebrospinal fluid (CSF), whole blood (ELISA) (Santiago et al., 2018). Uniquely, rRTPCR has shown to be proficient in detecting the presence of Zika virus in urine and amniotic fluid. Although all three viruses can be tested at the same time in a single rRT-PCR plate well (multiplex) using only one primer/probe set per well, the singleplex reaction option can test ZIKV in urine and amniotic fluid as well as ZIKV, CHIKV and DENV individually in other specimens.

Trioplex rRT-PCR targets the RNA for quantitative detection and differentiation among the ZIKV, CHIKV and DENV in human sera, whole blood and CSF (combined with a confirmed patient specific serum). It can also detect viral ZIKV RNA qualitatively in urine, and amniotic fluid..

While applied in specimens from patients of clinical symptoms and signs associated with ZIKV infections, or of the history of residence or travel in the active ZIKV endemic geographic regions, Trioplex rRT-PCR detects ZIKV in acute phage infection and even 14 days after the end of latent stage. Positive results indicate current infections, however negative results do not rule out possible ZIKV, CHIKV or DENV infections. Thus, it should not be used solely for the clinical treatment decision, and final diagnosis must take clinical symptoms, history and additional pathogenic information into consideration.

\subsubsection{Protocol Use Limitation}

Trioplex rRT-PCR has not been tested with massive clinical specimens, and can only be assayed under FDA's Emergency Use authorized laboratories. Distribution or modification of Trioplex rRT-PCR protocol is not allowed without the explicit CDC consent, limiting the widespread availability of test sites.

Trioplex rRT-PCR test can produce false-negative and false-positive results: false positives often results from cross contamination with previous DNA amplification; false-negatives may occur from contamination, timing of specimen collection ( during latent period or more than 14 days after the end of latent period) or failure to follow the authorized assay procedures.

\subsection{Serology IgM Antibody Detection: Anti-ZIKV IgM Antibody Capture Enzyme Linked Immunosorbent Assay (MAC ELISA)}

\subsubsection{Methodology Principle}

First Anti-IgM (the capture antibody) is coated on 96-well plates followed by the addition of patient serum, and then a known non-infectious viral antigen. An enzyme-conjugated anti-viral antibody is used to detect the presence of antigen. A chromogenic substrate interacts with the enzyme creating a colorimetric change is detected by a spectrophotometer (ELISA reader).

There are three types of anti-ZIKV IgM antibody capture enzyme linked immunosorbent assay (MAC-ELISA) responsible for initial serological screening of ZIKV infection: Centers for Disease Control and Prevention (CDC) Zika MAC-ELISA, the InBios ZIKV Detect ${ }^{\mathrm{TM}}$ MAC-ELISA, and the Euroimmun anti-Zika Virus IgM ELISA. Their test results are confirmed by additional confirmation of positive results on plaque reduction neutralization test (PRNT). 
Using specimen collected from possible ZIKV infection patients, the CDC and InBios MAC-ELISAs are mutually comparable with in test result agreements. However the Euroimmun ZIKV ELISA shows low positive agreement with either the CDC or InBios MAC-ELISAs. Furthermore, Euroimmun ZIKV ELISA did not show capability in distinguishing between ZIKV vs DENV in infected specimen. This presents an ongoing need and challenge to improve the specificity of these clinical assays in detecting ZIKV infection (Granger, Hilgart, Misner et al., 2017).

ZIKV is a single-stranded RNA virus that shares the overlapping RNA sequence with flaviviruses such as dengue, West Nile, Japanese encephalitis, and yellow fever (Faye, 2014; Hayes, 2009). Among these flavivirus, ZIKV and DENV have the most similar clinical cycles, symptoms and geographic distribution. Thus, DENV may induce enzyme-linked immunosorbent assay (ELISA) false positive results for ZIKV due to the crossreacting IgM antibodies against related flaviviruses. A confirmation test, the plaque reduction neutralization test (PRNT), must be used against various related flaviviruses to provide stronger evidence for the specificity of ZIKV true positive ELISA results (L'Huilier, 2017).

\subsection{PRNT (Plaque Reduction Neutralization)}

PRNT measures virus-specific neutralizing antibody titers, which is mainly IgG antibodies. Neutralizing antibodies against flavivirus infections can possibly persist for many years and provide lifelong immunity (Busch MP, 2008). Since in other primary flavivirus infection, PRNT is also used to identifying the flavivirus titer, the possibility of cross-reaction with other flavivirus can be ruled out with a neutralizing antibody titer larger or equal to 4-fold higher than previous titers. However 4-fold higher titer by PRNT might not definitely differentiate anti-Zika virus antibodies from the cross-reacting antibodies in the patients previously exposed to a related flavivirus either through infection or vaccination (Halstead, 1983; Johnson, 2005; Lindsey, 2017). For instance, a patient with history of previous DENV infection or yellow fever vaccination can present high titers of DENV or yellow fever induced IgG during later exposure to ZIKV (Halstead, 1983).

Because of the risk of fetal malformation in pregnant women infected with ZIKV, and the different clinical approach in managing the ZIKV and DENV infections, a careful and conservative interpretation of PRNT is suggested to reduce the misdiagnosis of either virus infections (Petersen, 2016).

The plaque reduction neutralization test (PRNT) is currently considered the gold standard of diagnostic tests in regards to ZIKV diagnosis (Shan et al., 2017). PRNT utilizes the infection plaques that are produced when virus containing samples are pipetted over mediated cells. Special media is spread to contain viral infection so that resulting plaques do not overlap and therefore discretely represent viral infection (World Health Organization, 2007). This is done with serial dilutions of the viral samples, and the resulting plaque counts are indication of reducing viral activity (WHO, 2007). While the test is considered reliable, it is labor intensive, requiring long wait time that can jeopardize patient's ability to access appropriate healthcare. In addition, PRNT is low throughput, inefficient, and high in labor, making it not the ideal diagnostic tool for detecting infected patients during rapid spreading epidemics (Shan et al., 2017).

\subsection{Newer Methods}

\subsubsection{Electrogenerated Chemiluminescence}

Electrogenerated Chemiluminescence (ECL) detects the light emission from the tested specimen undergoing high-energetic electron-transfer reactions between two electrodes. The emitted light can be captured by simple instruments making it an easy, low cost and ready-to-use device (Miao W, 2008). To amplify ECL detection signals, the polystyrene beads (PSBs) are further loaded with large amounts of water-insoluble homemade $\mathrm{Ru}(\mathrm{bpy}) 3[\mathrm{~B}(\mathrm{C} 6 \mathrm{~F} 5) 4] 2$ (bpy=2,2'-bipyridine) ECL labels. This increases ECL signals by several order of magnitudes (Miao \& Bard, 2004). By aggregating ECL label-loaded PSBs with anti-ZIKV antibody, a highly sensitive immunoassay was created for the detection and quantification of ZIKV viral particles in samples (Acharya, 2016).

\subsubsection{Reporter Virus Neutralization Test (RVNT)}

RVNT has proved to produce accurate and reliable readings for serum specimens (Shan et al., 2017). In addition, the lack of requiring invasive patient samples and ability for simultaneous high throughput testing allows it to surpass its traditional counterparts, even the PRNT, which has been considered the gold standard. However, RVNT has been limited to samples that do not contain hemoglobin, and more experimentation in comparing RVNT's performance against MAC-ELISA and PRNT for dual infected (ZIKV and DENV) patients is needed for confirming the validity of RVNT (Shan et al., 2017).

\section{Conclusion}

Though the ZIKV epidemic has been declared as no longer a global emergency, threats of its re-eruption still 
pose a threat to global health. Its close relation to other familial flavirus such as DENV, West Nile virus, and yellow fever virus, as well as its asymptomatic viremic stages creates challenges in correct diagnosis of patients with ZIKV infection. This paper explored the available diagnostic tools that have been currently proven to be able to detect ZIKV as well as the comparisons between the reliability and efficiency of aforementioned tests. It is evident that cross-reactivity is a important testing factor that a reliable diagnostic must be able to transcend due to ZIKV's molecular similarities to DENV. Furthermore, the rapidity in turn around rate for running diagnostics are also an important characteristic in order to efficiently track ZIKV spread and execute appropriate containing procedures as well as provide suitable medication to infected patients. More subtly, some tests are only exclusively compatible with a certain type of patient samples, such as blood, urine, or serum, which may pose a problem when only one type of sample is collectable from patients. Thus, the following factors are brought to light as important characteristics for a good ZIKV diagnostic tool: detection specificity, efficiency, and diverse sample compatibility.

New emerging tests from recent research, such as RVNT and ECL, are not as well known and widely used, are also discussed and compared. These tests yield promising results in cross-reactivity tests and turn-around rates. RVNT's high throughput performance provides promising prospects of large quantity simultaneous diagnosis that would greatly increase the efficiency and speed of ZIKV identification, treatment, and containment process. However, more experimentation is needed to improve and confirm the tests reliability and realize their limitations, if there are any. The grave implications of ZIKV and its underlying threat of eruption continues to spur new technological diagnostics.

\section{Competing Interests Statement}

The authors declare that there are no competing or potential conflicts of interest.

\section{References}

Acharya, D., Bastola, P., Le, L., Paul, A. M., Fernandez, E., Diamond, M., ... Bai, F. (2016). An Ultrasensitive Electrogenerated Chemiluminescence-based Immunoassay for Specific Detection of Zika Virus Scientific Reports 2016,6,32227. https://doi.org/10.1038/srep32227

Busch, M. P., Kleinman, S. H., Tobler, L. H., Kamel, H. T., Norris, P. J., Walsh, I., ... \& Linnen, J. M. (2008). Virus and antibody dynamics in acute West Nile virus infection. The Journal of infectious diseases, 198(7), 984-993. https://doi.org/10.1086/591467

Centers for Disease Control and Prevention. Diagnostic Tests for Zika Virus. (Feb. 2016). Retrievedfrom https://www.cdc.gov/zika/hc-providers/types-of-tests.html

Dick, G. W. A., Kitchen, S. F., \& Haddow, A. J. (1952). Zika virus (I). Isolations and serological specificity. Transactions of the royal society of tropical medicine and hygiene, 46(5), 509-520. https://doi.org/10.1016/0035-9203(52)90042-4

Duffy, M. R., Chen, T. H., Hancock, W. T., Powers, A. M., Kool, J. L., Lanciotti, R. S., ... \& Guillaumot, L. (2009). Zika virus outbreak on Yap Island, federated states of Micronesia. New England Journal of Medicine, 360(24), 2536-2543. https://doi.org/10.1056/NEJMoa0805715

Faye, O., Freire, C. C., Iamarino, A., Faye, O., de Oliveira, J. V. C., Diallo, M., \& Zanotto, P. M. (2014). Molecular evolution of Zika virus during its emergence in the 20th century. PLoS neglected tropical diseases, 8(1), e2636. https://doi.org/10.1371/journal.pntd.0002636

Granger, D., Hilgart, H., Misner, L., Christensen, J., Bistodeau, S., Palm, J., ... \& Theel, E. S. (2017). Serologic testing for Zika virus: comparison of three Zika virus IgM-screening enzyme-linked immunosorbent assays and initial laboratory experiences. Journal of clinical microbiology, 55(7), 2127-2136. https://doi.org/10.1128/JCM.00580-17

Haddow, A. J., Williams, M. C., Woodall, J. P., Simpson, D. I. H., \& Goma, L. K. H. (1964). Twelve isolations of Zika virus from Aedes (Stegomyia) africanus (Theobald) taken in and above a Uganda forest. Bulletin of the World Health Organization, 31(1), 57.

Halstead, S. B., Rojanasuphot, S., \& Sangkawibha, N. (1983). Original antigenic sin in dengue. The American journal of tropical medicine and hygiene, 32(1), 154-156. https://doi.org/10.4269/ajtmh.1983.32.154

Hayes, E. B. (2009). Zika virus outside Africa. Emerging infectious diseases, 15(9), 1347. https://doi.org/10.3201/eid1509.090442

Johnson, B. W., Kosoy, O., Martin, D. A., Noga, A. J., Russell, B. J., Johnson, A. A., \& Petersen, L. R. (2005). West Nile virus infection and serologic response among persons previously vaccinated against yellow fever and Japanese encephalitis viruses. Vector-Borne \& Zoonotic Diseases, 5(2), 137-145. 
https://doi.org/10.1089/vbz.2005.5.137

Kwong, J. C., Druce, J. D., \& Leder, K. (2013). Zika virus infection acquired during brief travel to Indonesia. The American journal of tropical medicine and hygiene, 89(3), 516-517. https://doi.org/10.4269/ajtmh.130029

L'Huillier, A. G., Hamid-Allie, A., Kristjanson, E., Papageorgiou, L., Hung, S., Wong, C. F., ... \& Drebot, M. (2017). Evaluation of Euroimmun Anti-Zika virus ELISAs (IgM \& IgG) for Zika virus serologic testing. Journal of clinical microbiology, JCM-00442.

Lindsey, N. P., Staples, J. E., Powell, K., Rabe, I. B., Fischer, M., Powers, A. M., ... \& Hancock, W. T. (2018). Ability to serologically confirm recent Zika virus infection in areas with varying past incidence of dengue virus infection in the United States and US territories in 2016. Journal of clinical microbiology, 56(1), e01115-17. https//doi.org/10.1128/JCM.01115-17

Miao, W. (2008). Electrogenerated chemiluminescence and its biorelated applications. Chemical reviews, 108(7), 2506-2553. https://doi.org/10.1021/cr068083a

Miao, W., \& Bard, A. J. (2004). Electrogenerated chemiluminescence. 80. C-reactive protein determination at high amplification with [Ru (bpy) 3] 2+-containing microspheres. Analytical chemistry, 76(23), 7109-7113. https://doi.org/10.1021/ac048782s

Oduyebo, T. (2016). Update: interim guidelines for health care providers caring for pregnant women and women of reproductive age with possible Zika virus exposure-United States, 2016. MMWR. Morbidity and mortality weekly report, 65 . https://doi.org/10.15585/mmwr.mm6512e2

Wong, P. S. J., Li, M. Z. I., Chong, C. S., Ng, L. C., \& Tan, C. H. (2013). Aedes (Stegomyia) albopictus (Skuse): a potential vector of Zika virus in Singapore. PLoS neglected tropical diseases, 7(8), e2348. https://doi.org/10.1371/journal.pntd.0002348

Santiago, G. A., Vázquez, J., Courtney, S., Matías, K. Y., Andersen, L. E., Colón, C., ... \& Muñoz-Jordan, J. L. (2018). Performance of the Trioplex real-time RT-PCR assay for detection of Zika, dengue, and chikungunya viruses. Nature communications, 9(1), 1391. https://doi.org/ 10.1038/s41467-018-03772-1

Shan, C., Ortiz, D. A., Yang, Y., Wong, S. J., Kramer, L. D., Shi, P. Y., ... \& Ren, P. (2017). Evaluation of a novel reporter virus neutralization test for the serological diagnosis of Zika and dengue virus infection. Journal of clinical microbiology, JCM-00975. https://doi.org/10.1128/JCM.00975-17

World Health Organization [WHO]. (2016). Zika Virus. Retrieved 6 September, 2016, from http://www.who.int/mediacentre/factsheets/zika/en/

World Health Organization [WHO]. (2007). Guidelines for Plaque Reduction Neutralization Testing of Human Antibodies to Dengue Viruses. Initiative for Vaccine Research. World Health Organization. Retrieved from http://www.who.int/mediacentre/factsheets/zika/en/

\section{Copyrights}

Copyright for this article is retained by the author(s), with first publication rights granted to the journal.

This is an open-access article distributed under the terms and conditions of the Creative Commons Attribution license (http://creativecommons.org/licenses/by/4.0/). 